\title{
La participación de las mujeres en radios comunitarias en India frente al silenciamiento sistémico y la brecha digital
}

\author{
Amaia LANDABURU \\ Universidad del País Vasco/Euskal Herriko Unibertsitatea \\ amaia.landaburu@ehu.eus \\ https://orcid.org/0000-0002-0014-9054
}

\section{The participation of women in community radios in India against systemic silence and the digital divide}

\begin{abstract}
RESUMEN
Este artículo analiza la participación de las mujeres como grupo subalterno en tres experiencias de radios comunitarias en India. Se centra en los procesos de transformación iniciados por ellas, con un acercamiento a las problemáticas que enfrentan, así como un estudio de su papel como comunicadoras y referentes en contextos de desigualdad y discriminación extremas.

El reconocido Informe MacBride (1980) recogía entre sus reivindicaciones la necesidad de una comunicación más democrática. En esta línea, la democratización mediática es la principal preocupación de esta investigación que parte, a su vez, de la pregunta planteada por Spivak (1998): “¿Puede hablar el sujeto subalterno?".

Presentamos los resultados de un análisis cualitativo mediante técnicas como la entrevista personal y la observación participante. Analizamos varios relatos de experiencias de mujeres trabajadoras de radios comunitarias en India, con el fin de reflejar la contribución de la comunicación participativa a la realización del derecho a la comunicación en India.
\end{abstract}

PALABRAS CLAVE

Comunicación participativa; Radio comunitaria; Perspectiva de género; Democratización mediática; Brecha digital.

\section{ABSTRACT}

This article analyses the participation of women as a subaltern group in three community radio experiences in India. It focuses on the transformation processes initiated by them, with an approach to the problems they face, as well as a study of the role they play as communicators and role models in contexts of extreme inequality and discrimination. The renowned MacBride Report (1980) included the need for more democratic communication among its claims. In this regard, media democratisation is the main concern of this research, which sets out from the question posed by Spivak (1998): "Can the Subaltern speak?".

The paper presents the results of a qualitative analysis including personal interviews and participant observation. In this work we analyse experiences of women workers in three community radios initiatives in India, with the aim of reflecting the contribution of Participatory Communication to the realisation of Communications Rights in India.

KEYWORDS

Participatory communication; Community radio; Gender perspective; Media democratisation; Digital divide. 


\section{Introducción}

Desde la perspectiva crítica que insiste en la necesidad de incluir la participación como elemento esencial en los procesos de la comunicación, este trabajo pretende contribuir a la reivindicación del derecho a la comunicación partiendo de las bases sentadas en el Informe MacBride (1980). La investigación se centra en las experiencias de las mujeres subalternas como participantes de las radios comunitarias en India, en su capacidad de acción (Spivak, 1998; Dutta, 2012), y está guiada por la necesidad de una democratización mediática que pueda garantizar el derecho a la comunicación de las voces silenciadas, en especial, de las poblaciones campesinas, tribales y de las periferias urbanas.

Esta investigación toma como punto de partida las ideas de Freire (1970), desde su propuesta teórica se enfoca el interés de la investigación en los procesos y en la acción dialógica de las poblaciones silenciadas y excluidas, mediante los que han sido capaces de transformar su realidad social y construir una diferente, y se aborda la documentación y análisis de experiencias de comunicación participativa para tratar de presentar aprendizajes desde la práctica. En su pedagogía crítica, Freire (1970) destacó la importancia del diálogo en la investigación, en la creación de conocimiento a partir de los sujetos, perspectiva en la que se enmarca este trabajo.

Seguimos las recomendaciones de autoras de referencia en el estudio de la comunicación participativa, de abordar la investigación del derecho a la comunicación desde el ángulo de "la comunicación de los segmentos subalternos organizados de la sociedad" (Krohling Peruzzo, 2016:11). A la vez que entendemos las radios comunitarias como unos medios participativos que "son evidencias del ejercicio efectivo del derecho a la comunicación" (Ibid., p. 17).

Desde la academia en India, las investigaciones de referencia en el estudio de la comunicación participativa, y que tienen en cuenta la perspectiva de género, consideran que la participación de la comunidad es un factor intrínseco al concepto de medios comunitarios (Malik, 2015), haciendo referencia a la gestión, a la producción de contenido y a la vida comunitaria en torno a estos medios.

En el análisis de experiencias, hemos incluido la perspectiva de género tomando como referencia investigaciones que han reflexionado en torno a la dimensión transformadora de la comunicación, incluyendo en sus investigaciones análisis sobre la participación y el empoderamiento de las mujeres con estudios de caso sobre radios comunitarias en India (Pavarala y Malik, 2007; Malik y Bandelli, 2012).

Este artículo recoge aprendizajes de los procesos de comunicación iniciados en poblaciones subalternas, en concreto por mujeres comunicadoras de radios comunitarias en India, frente al silenciamiento sistémico y la brecha digital, que trazan un camino de la exclusión social a la expresión popular. Se basa en la idea de que las acciones, críticas y cuestionamientos que surgen de los movimientos sociales contra la discriminación y las desigualdades, o por la democratización de los recursos y la justicia social, reflejan una profundización de la democracia (Mohanty, 2010).

Del mismo modo este trabajo parte de la idea de que el acceso al poder cambia continuamente en las comunidades y entiende los medios comunitarios como experiencias de comunicación en las que las participantes se encuentran en un proceso de continua renegociación con el entorno excluyente, enfocando el interés de la investigación en comprender cómo se transforman las múltiples relaciones de poder en la vida cotidiana (Rodriguez, 2001). Desde este marco conceptual, la democratización de la comunicación es entendida como un proceso complejo en el que los medios comunitarios pueden contribuir a transformar el acceso al poder de las participantes en sus respectivas comunidades (Ibid.).

Desde un enfoque que pone el énfasis en los procesos de transformación de las personas y comunidades, entendemos los medios comunitarios como alimentadores de esos procesos cuya práctica de la comunicación emprende el camino del diálogo y la participación (Kaplún, 2002). En concreto, la investigación intenta conocer experiencias concretas de ese momento significativo "en que los participantes quiebran su dilatada "cultura del silencio" y comienzan a recuperar la palabra" (Kaplún, 2002:138).

\subsection{El silenciamiento sistémico en India}

En el panorama mediático actual en India, las poblaciones subalternas sufren un silenciamiento sistémico que es resultado de unas dinámicas de exclusión que, sobre todo, afectan a poblaciones rurales campesinas, dalits, adivasis y a las mujeres. Así, sus voces quedan silenciadas e ignoradas, una problemática que ha sido analizada en varias investigaciones de referencia (Jeffrey, 2001; Skuse et al., 2007; Tacchi et al., 2009; Tacchi, 2009; Drèze y Sen, 2013; Mander, 2015). Mander (2015) denomina a esta situación "un exilio de los pobres" y subraya que la normativización de la desigualdad en India es resultado de varios sistemas normativos: el sistema de castas, el sistema de clases heredado de los colonizadores británicos y el neoliberalismo. Esto resulta en una exclusión múltiple que es, por tanto, sistémica y afecta a muchos ámbitos de la vida, incluida la exclusión mediática. Mientras, los medios dominantes y elitistas refuerzan el statu quo y miran hacia otro lado ante esta situación de exclusión múltiple y frente a las desigualdades endémicas (Drèze y Sen, 2013; Mander, 2015).

Los prejuicios, sesgos y enfoques selectivos en los medios dominantes en India han sido señalados por Drèze y Sen (2013); en consecuencia, los problemas de desigualdad quedan escasamente reflejados. A esto se suma una ausencia 
de dalits en los puestos de reporteros, editores o puestos de gestión (Jeffrey, 2001) y el prejuicio de los profesionales de los medios que provienen en su mayoría de las clases y castas altas de esta sociedad (Drèze y Sen, 2013).

De este modo, los medios alineados con las esferas de poder político y económico, fomentan un silenciamiento sistémico de las poblaciones más vulnerables, reforzado por una falta de interés tanto de los medios como de las clases medias indias por las necesidades e intereses de los excluidos y las minorías (Drèze y Sen, 2013; Thomas, 2014).

\subsection{La brecha digital en India}

Frente a la imagen de India como centro neurálgico de las NTIC y de fuerza económica emergente en el escenario mundial, encontramos un problema de acceso a Internet y una brecha digital, debido a la desigualdad y discriminación extremas.

En 2019 el 60\% de la población india carecía de acceso a Internet. Esta desigualdad en el acceso afecta notablemente a las mujeres, siendo entre los usuarios y las usuarias con acceso a internet el 65\% hombres y el 35\% mujeres (IAMAI-Nielsen, 2019), lo que refleja además una brecha digital de género en el acceso a Internet en este contexto excluyente.

La desigualdad de género queda reflejada igualmente en los datos oficiales relativos al acceso a los medios en general. El porcentaje de mujeres sin acceso habitual a los medios tradicionales (televisión, radio, prensa, cine) es del 35\% frente al 18\% de los hombres (Kishor y Gupta, 2009).

Además, debemos tener en cuenta otros aspectos de este contexto en relación a la discriminación que sufren las mujeres en este país, con resultados como el del informe de la fundación Thomson Reuters (2018) en el que India fue clasificada como el país más peligroso para las mujeres.

La problemática de la desigualdad de género en la expansión de las TIC en India ha sido analizada por Pande (2012), quien señala que esta ha resultado en una brecha digital de género, y en una desigualdad en términos de acceso, alfabetización digital y seguridad.

En India, la capacidad de las mujeres para participar en las Tecnologías de la Información está determinada por el bajo estatus atribuido a las mujeres y niñas en la sociedad india, y la pobreza extrema y una infraestructura deficiente que limita el acceso de las mujeres a la educación y a las tecnologías de la información (Pande, 2012. p.289).

Las problemáticas evidenciadas en este contexto hacen que sea necesario investigar las alternativas que representan una oportunidad para la realización del derecho a la comu- nicación de las poblaciones excluidas por la brecha digital de género. De este modo, vemos necesario considerar la exclusión como factor esencial en la investigación, tal y como ha señalado Aruna Roy (2016). Teniendo en cuenta que incorporar la perspectiva de género, como han remarcado Malik y Bandelli (2012), es significativo en este contexto donde las mujeres están excluidas de la esfera pública.

La contribución de los medios comunitarios a transformar esta realidad ha sido igualmente de interés para la investigación en comunicación participativa en India (Pande, 2012; Malik, 2012), tanto como solución a la exclusión y silenciamiento sistémico, como oportunidad para las voces silenciadas de formar parte del movimiento por la democratización mediática y el derecho a la comunicación.

\subsection{La Comunicación Participativa para el empoderamiento en India}

Las investigaciones que han analizado la participación de las mujeres en los medios comunitarios en India desde la perspectiva de la comunicación para el empoderamiento (Naqvi, 2007; Pavarala y Malik, 2007, S. Chatterji, 2010; Thomas, 2011; Malik y Bandelli, 2012; Yalala, 2015; Moitra y Kumar, 2016) han demostrado que los medios comunitarios pueden contribuir a la transformación de las vidas de las mujeres. Son trabajos de referencia que indagan en las articulaciones entre la desigualdad social y los medios, así como la contribución de la radio a la "amplificación de la voz" de las mujeres (Moitra y Kumar, 2016).

Este trabajo también se basa en los hallazgos de investigaciones sobre comunicación participativa realizada en la India, como la de Malik y Bandelli (2012) que analiza el papel de las mujeres participantes de dos radios comunitarias indias, Sangham Radio y Radio Namaskar. De estas investigaciones aprendemos que son muchas, las problemáticas que enfrentan las mujeres por las jerarquías sociales existentes y la discriminación de género, tanto como oyentes como reporteras de estas radios (Ibid.). La participación de las mujeres en estas iniciativas es, por tanto, significativa y estas investigaciones representan una búsqueda de respuestas a la necesidad de amplificar la voz con el empoderamiento de las poblaciones excluidas y la capacidad de acción de los grupos subalternos.

\subsection{Objetivos de la investigación}

Las iniciativas de las radios comunitarias del análisis pretenden contribuir al derecho a la comunicación de las poblaciones silenciadas y, de este modo, suplir el vacío comunicativo existente como consecuencia del silenciamiento sistémico y la brecha digital. El objetivo general de la investigación está formulado a partir de la siguiente pregunta: ¿Qué aprendizajes podemos inferir desde estas experiencias de comunicación? 
Analizaremos estas experiencias con el fin de reflejar la contribución de la comunicación participativa a la realización del derecho a la comunicación. Los objetivos de la investigación son, por un lado, documentar ejemplos de buenas prácticas de comunicación participativa que están involucrando a poblaciones silenciadas; por otro, profundizar en el enfoque de género a partir de las experiencias de las mujeres participantes en los medios comunitarios y conocer los procesos que han iniciado para romper ese silencio; y, por último, intentar conocer las estrategias de participación con las que estas iniciativas radiofónicas dan respuesta a las problemáticas del silencio y la exclusión, así como identificar factores que contribuyen y factores que dificultan la realización del derecho a la comunicación de las participantes.

\section{Materialy métodos}

La metodología de la investigación está basada en un trabajo previo de la Asociación Mundial de Radios Comunitarias y la Women's International Network en la región de Asia Pacífico (AMARC-WIN Asia-Pacific, 2013). Se trata de un informe comparativo que identifica y evalúa aspectos clave de la participación de las mujeres en las radios comunitarias de varios países de esta región, basándose en los principios de las Políticas de Género para la Radios Comunitarias de estas asociaciones (AMARC-WIN, 2008).

En este trabajo seguimos una metodología cualitativa mediante las técnicas de la observación participante y entrevistas personales semiestructuradas realizadas en 2016 a tres participantes representando a las radios comunitarias Gurgaon Ki Awaaz, Radio Brahmaputra y Radio Bundelkhand durante las visitas a las iniciativas radiofónicas. Las entrevistadas forman parte de las iniciativas tanto en la dirección de la emisora como en la producción.

El material del análisis se compone, por tanto, de las transcripciones de tres entrevistas realizadas durante las visitas a las emisoras y de las notas del diario de campo con información recabada de la observación participante, así como de la documentación fotográfica realizada y de materiales informativos proporcionados desde las mismas emisoras.

Las entrevistas estuvieron guiadas en base a los principios de las Políticas de Género para las Radios Comunitarias (AMARC WIN, 2008) anteriormente mencionados. A partir de dicho documento, elaboramos los puntos a comentar.

Las radios seleccionadas para el estudio, siguiendo las recomendaciones de investigadores y activistas del movimiento de radios comunitarias en India, abordan las problemáticas de las mujeres en las comunidades y conciben el enfoque de género como parte de sus valores. La participación de la comunidad es entendida como esencial en el proceso de la comunicación de las iniciativas estudiadas; tanto en la producción de los programas radiofónicos, que en todos los casos está en manos de personas que provienen de las mismas comunidades a las que dan servicio, como en la gestión igualmente relevante para estas emisoras, pues solo así pueden conocer de primera mano las necesidades, intereses y problemáticas que son relevantes en sus comunidades.

\subsection{Radio Brahmaputra}

Radio Brahmaputra está ubicada en Dibrugarh, Assam, en la región fronteriza del Noreste de India, a orillas del río Brahmaputra y cerca de los Tea States (plantaciones de té) característicos de esta región. Dibrugarh es conocida como la "Tea City of India" y sus plantaciones reflejan el expolio colonial que desde el siglo XIX provocó el despojo de los bosques, la reducción de las tierras comunales y la esclavitud de las poblaciones trabajadoras en la industria del té. Desde la época colonial estos cultivos están orientados a la exportación y su producción está actualmente en manos de grandes empresas británicas y entramados comerciales nacionales.

Radio Brahmaputra es la primera radio comunitaria en el noreste de India que llega a zonas rurales. Como plataforma local, proporciona un espacio en el que poder compartir información y conocimientos con el fin de poder transformar las injusticias sociales en condiciones dignas, e iniciar procesos de apropiación mediante la participación de las comunidades para que puedan expresarse y acceder a la información que necesitan.

La emisora da servicio a las comunidades adivasi trabajadoras de las plantaciones de té, que viven en condiciones de exclusión y de explotación por la industria del té. Son, en su mayoría, tribus originarias de otros Estados centrales de India que migraron durante la colonización y el idioma mayormente hablado en estas poblaciones es el sadri, utilizado por la radio, además de los idiomas assamese (la lengua oficial del Estado de Assam), bhojpuri, mishing y bodo. La radio emite en varios idiomas gracias a un equipo de personas que provienen de las comunidades a las que la radio da servicio y a una red de voluntarias y voluntarios con las que organizan talleres de formación, donde los y las participantes pueden utilizar la radio y producir contenido para los programas de acuerdo a los intereses y necesidades de sus propias comunidades.

La radio también se dirige a las poblaciones que viven en los slums de las zonas urbanas y a las comunidades de las islas del río Brahmaputra quienes, como consecuencia del cambio climático, enfrentan el problema de la erosión del suelo en sus territorios por la acción del agua del caudal que está haciendo desaparecer sus tierras, un fenómeno que se ha visto agravado en los últimos años. Desde la radio suelen desplazarse periódicamente a estas islas para realizar grabaciones en terreno, y registrar aportaciones, canciones populares e historias locales en sus propios idiomas con el fin de motivar su 


\begin{tabular}{|l|l|l|l|}
\hline \multicolumn{1}{|c|}{ Nombre } & Desde & \multicolumn{1}{|c|}{ Lugar } & \multicolumn{1}{c|}{ Objetivos } \\
\hline $\begin{array}{l}\text { Radio } \\
\text { Bundelkhand }\end{array}$ & 2008 & $\begin{array}{l}\text { Orchha, } \\
\text { Madhya } \\
\text { Pradesh }\end{array}$ & $\begin{array}{l}\text { Dar voz a las personas sin voz en la comunidad. Dar servicio a la comunidad } \\
\text { con programas de interés local. Empoderamiento de las comunidades, en } \\
\text { especial de las mujeres, las poblaciones jóvenes y los grupos marginados. }\end{array}$ \\
\hline $\begin{array}{l}\text { Gurgaon } \\
\text { ki Awaaz }\end{array}$ & 2009 & $\begin{array}{l}\text { Gurgaon } \\
\text { Gurugram), } \\
\text { Haryana }\end{array}$ & $\begin{array}{l}\text { Proporcionar una plataforma en la que la comunidad local pueda } \\
\text { tener acceso a información relevante, y como espacio en el que } \\
\text { poder expresar su cultura, intereses y preocupaciones cotidia- } \\
\text { nas. Radio enfocada en las comunidades migrantes. }\end{array}$ \\
\hline $\begin{array}{l}\text { Radio } \\
\text { Brahmaputra }\end{array}$ & 2015 & $\begin{array}{l}\text { Fortalecer la participación de las personas y contribuir a que las comunidades } \\
\text { Dibrugarh, } \\
\text { Assam }\end{array}$ & $\begin{array}{l}\text { más vulnerables obtengan un mayor acceso a la justicia, la dignidad y los servi- } \\
\text { cios básicos. Mediante información y conocimiento sobre el medioambiente, la } \\
\text { salud, la agricultura, los medios de vida, recursos como el agua, la educación, el } \\
\text { empoderamiento de las mujeres, la cultura local y las artes populares. }\end{array}$ \\
\hline
\end{tabular}

Tabla 1. Resumen de las iniciativas del análisis. Fuente: páginas web de los proyectos, trabajo de campo, el directorio web de radios comunitarias de One World Foundation India (2016). Elaboración propia.

participación y reflejar en su programación la diversidad lingüística local y la diversidad cultural de este territorio en el que conviven diferentes comunidades.

\subsection{Radio Bundelkhand}

Radio Bundelkhand está ubicada en una zona rural de la India central y fue una de las primeras radios del Tercer Sector en India. La emisora forma parte de un campus de proyectos dedicados a innovaciones y tecnologías limpias de desarrollo alternativas, denominado TARAgram y establecido por la ONG Development Alternatives (DevAlt).

La región de Bundelkhand donde se ubica la radio sufre graves sequías cada año. El cambio climático afecta directamente a las comunidades campesinas de las zonas rurales que conforman esta región central de India, donde los suministros de agua son escasos y algunas poblaciones dependen de bombas de agua manuales.

La radio comunitaria está centrada en las temáticas de la agricultura, el cambio climático, la salud, la gobernanza local y los procesos de empoderamiento de las comunidades rurales de esta región.

El equipo de la radio está conformado por personas de las comunidades a las que se dirige la radio y quienes se unieron al proyecto al conocer esta iniciativa impulsada desde la ONG DevAlt en la que poder expresarse en su lengua local, el bundeli. La radio también cuenta con la colaboración de artistas locales que han encontrado en la emisora una plataforma en la que poder darse a conocer y contribuir con sus canciones populares, teatro y música local.

La radio tiene establecidas colaboraciones con otras emisoras comunitarias de la región de Bundelkhand para la creación de contenidos, formación y producción de programas sobre temáticas de interés común, en torno a los asuntos relativos a la tierra, el cambio climático y los medios de subsistencia.

\subsection{Gurgaon ki Awaaz}

La radio comunitaria Gurgaon ki Awaaz está situada en Gurgaon (Gurugram), ciudad satélite a treinta kilómetros de la capital india que ha sido recientemente y en poco tiempo convertida en un centro empresarial y comercial. Sus infraestructuras y el sector inmobiliario han crecido rápidamente con una escasa planificación. La rápida expansión de la urbanización ha dado lugar a una ciudad en la que los barrios marginales colindan con centros y oficinas comerciales.



Radios comunitarias:

Radio Bundelkhand

Radio Brahmaputra

Gurgaon Ki Awaaz

Figura 1. Localización de las radios comunitarias. Elaboración propia con la herramienta Google MyMaps. 
En estas zonas urbanas periféricas, las comunidades locales han ido conformándose en procesos de reasentamiento. Gurgaon es un destino principal del éxodo rural proveniente de otros estados centrales de India, como Uttar Pradesh o Bihar; una ciudad que ha atraído a poblaciones provenientes de zonas rurales en busca de medios de vida, que han contribuido con su mano de obra y han sido al mismo tiempo marginadas en el proceso de urbanización de la ciudad; sus necesidades han sido generalmente ignoradas en asuntos como los servicios sociales o de infraestructuras.

La radio fue establecida por la ONG TRF en 2009 como una plataforma en la que las comunidades migrantes y obreras puedan expresarse. La emisora también intenta dar respuesta a las problemáticas asociadas a la alienación en las grandes ciudades, mediante una programación que incluye la cultura tradicional y los idiomas locales de las poblaciones migrantes.

La radio cuenta con una notable participación de mujeres en su equipo, así como una extensa red de voluntariado.

\section{Análisis y resultados}

A partir de las experiencias recogidas en el estudio, este apartado intenta en primer lugar documentar y describir ejemplos de buenas prácticas de radios comunitarias que intentan contribuir al derecho a la comunicación de las poblaciones silenciadas y abordar las problemáticas de las mujeres como grupo subalterno; entre otras, la exclusión por motivos de género, la brecha digital y el silenciamiento sistémico. A continuación, con el objetivo de profundizar en el enfoque de género, hemos intentado estudiar los procesos de transformación iniciados por las mujeres participantes de las radios comunitarias para romper el silenciamiento sistémico mediante su participación en las iniciativas radiofónicas, su paso de voces silenciadas a participantes y comunicadoras y su papel como referentes en sus comunidades. En este punto, enfocamos el interés de la investigación en comprender cómo cambian las múltiples relaciones de poder en la vida cotidiana y qué procesos de deconstrucción y reconstrucción de la identidad tienen lugar en estas experiencias comunicativas (Rodriguez, 2001). Finalmente, intentamos conocer las estrategias de participación de las iniciativas radiofónicas, así como los factores que han contribuido y los que han obstaculizado los procesos de acceso y apropiación de estos medios comunitarios.

\subsection{Las radios comunitarias como entornos para una participación igualitaria}

Desde las iniciativas analizadas consideran que la participación de las mujeres en los equipos de trabajo es igualitaria. De acuerdo a sus experiencias, organizan el trabajo desde un enfoque colaborativo y con una gran flexibilidad en las funciones y tareas a desempeñar en un entorno laboral en el que prima el trabajo en equipo y comparten funciones, considerándolo como un proceso de aprendizaje dentro del funcionamiento de la radio en el que las mujeres participan y tienen un gran peso tanto en la producción como en la gestión. De los tres casos de estudio, destaca la emisora Gurgaon ki Awaaz por una mayoría de mujeres participantes. Desde la emisora explicaron los factores que habían contribuido a ello: "las mujeres se quedan por el ambiente de trabajo seguro que brinda la radio, y les gusta tener voz aquí, poder conversar sobre sus propias historias, con sus propias narrativas, y sobre los problemas que les conciernen". Las entrevistadas confirman que las radios son un entorno seguro y tienen un ambiente de trabajo en el que no sienten miedo, tampoco, aunque sean las primeras en llegar a horas tempranas o cuando hay poca gente.

La flexibilidad en el horario de emisión para poder adaptarse a las necesidades y disponibilidad horarias de las trabajadoras, así como la no exigencia en la realización de trabajo que puedan comportar riesgos para las reporteras en referencia a las grabaciones en exterior y a horarios nocturnos, favorece la participación de las mujeres. En las emisoras tienen en cuenta la inseguridad para las mujeres en este contexto y asignan tareas en las que no tengan que hacer frente a estos riesgos, lo que supone por otro lado un mayor peso de las emisiones y grabaciones en el estudio y que no ofrezcan emisiones nocturnas.

En cuanto al uso de la tecnología por parte de las mujeres en los equipos de las radios, afirman desde Radio Bundelkhand que se desenvuelven fácilmente, puesto que han recibido una formación técnica adecuada y tienen las capacidades técnicas necesarias para llevar adelante la radio. "Tenemos las mismas oportunidades de usar la tecnología, estamos usando cualquier tecnología que use el personal masculino: ordenador, edición, mixer, todo. Si la voz no va, sabemos cuál es el problema. Lo que pasa aquí es que todos tienen el mismo rol. No hay prejuicios de género. Lo que sea que tengan que hacer, nosotras tenemos que hacer el mismo trabajo.", se recoge en una de las respuestas.

En referencia a las condiciones laborales, los salarios son bajos y las condiciones laborales son poco estables. Las iniciativas se apoyan en gran medida en el trabajo voluntario, lo que hace que decaiga el interés en participar entre los hombres participantes, según explican en Gurgaon ki Awaaz, dado que tradicionalmente son los que con su trabajo generan la mayor fuente de ingresos familiar. En ninguna de las entrevistas mencionaron expresamente disponer de una política de personal y salarial definida para promover una mayor participación de las mujeres. No obstante, sí confirman disponer de una política contra el acoso sexual. 


\subsection{El papel de las mujeres participantes de las radios comunitarias}

En este punto intentamos documentar y analizar el papel desempeñado por las mujeres participantes como referentes en la comunidad y dentro del equipo de las radios comunitarias, quienes como mujeres y como poblaciones subalternas experimentan una exclusión sistémica del acceso a los recursos y unas relaciones de poder en las comunidades en las que trabajan.

La discriminación de las mujeres como problemática del contexto y entorno próximo es un factor que obstaculiza notablemente la participación de las mujeres en las radios comunitarias. Desde las radios aseguran haber iniciado un proceso de concienciación, sin embargo, transformar esa realidad discriminatoria en la comunidad es un proceso mucho más complejo. Desde Radio Brahmaputra expresan preocupación por las limitaciones existentes frente a un problema de discriminación estructural, mientras que destacan la importancia que tiene la participación de las mujeres como reporteras y locutoras para la comunidad y para la audiencia; lo valoran como algo indispensable para la representación e identificación de las audiencias con la radio.

El papel desempeñado por las reporteras como referentes en sus propias comunidades y los procesos de transformación y empoderamiento iniciados son una contribución a transformar las relaciones de poder existentes en una realidad excluyente con las mujeres y los grupos subalternos.

Desde la emisora de Radio Bundelkhand explican que las mujeres enfrentan muchas limitaciones en sus comunidades y familias. La experiencia personal de la reportera entrevistada ilustra estas dificultades y el proceso de transformación iniciado con su participación en la radio. La participante experimentó una fuerte oposición de sus suegros, que forman parte de su unidad convivencial (como es habitual en India donde los hogares suelen estar conformados por una familia extensa), cuando expresó su interés de formar parte del equipo de la radio y su decisión de trabajar como reportera. Tras un proceso de negociación fueron entendiendo la relevancia de esta decisión que finalmente respetaron. La reportera expresa una gran motivación por este trabajo, por las siguiente razones que explica: "Es por el respeto que la gente da, es un vínculo con la radio y la gente que trabaja aquí y los que están escuchando".

La comunicadora ha pasado de ser silenciada a ser reportera de los asuntos de interés en su comunidad, proporcionando información necesaria y relevante, así como haciendo crecer la seguridad en sí misma; dando lugar a los procesos de deconstrucción y reconstrucción de la identidad y de empoderamiento expuestos por Rodriguez (2001). Su papel como referente en la comunidad es relevante, puesto que con sus experiencias cotidianas de acción dialógica ha transformado una realidad excluyente.

Estas evidencias empíricas muestran resultados que coinciden con investigaciones de referencia llevadas a cabo en otros contextos acerca de contribuciones similares a la realización del derecho a la comunicación; como el trabajo de referencia de Rodriguez (2001), quien observa que las experiencias de comunicación analizadas son "Diferentes formas en las que los medios participativos pueden convertirse en lugares de empoderamiento de las mujeres. (...) [y] "... procesos que aumentan la confianza en sí mismas de las mujeres que han sido tradicionalmente silenciadas." (Rodriguez, 2001: 123); y observa cómo esta apropiación "puede provocar procesos transformadores que se extienden en un efecto dominó a través de muchas capas del tejido social de una comunidad" (Ibid. p. 127).

\subsection{Las estrategias de participación}

La accesibilidad de la comunidad como audiencias participantes en los procesos de comunicación es muy significativa para las iniciativas radiofónicas, estas son concebidas como espacios abiertos a todas las personas e intentan ser inclusivas para que las mujeres participen desde la igualdad. Tal y como explican desde Radio Brahmaputra: "La radio está completamente a cargo de la gente de la comunidad, por lo que es una plataforma muy fácil y accesible, y es popular entre la comunidad".

Las grabaciones en terreno, las llamadas de los oyentes a la radio, la participación de oyentes de la comunidad en el estudio de grabación, las entrevistas y los talleres de formación son las prácticas de participación más habituales en las radios estudiadas.

Algunas de las emisoras comenzaron a emitir programas en narrowcasting y es una de las actividades que continúa organizando Radio Brahmaputra entre grupos de mujeres con el fin de iniciar un diálogo con las comunidades excluidas a las que dirigen sus programas y con la que intentan dar respuesta a este déficit de acceso a los medios.

A partir de los datos empíricos, la radio comunitaria prueba ser un medio accesible que intenta dar respuesta a la problemática de la brecha digital, evidente en las zonas rurales donde existen escasas infraestructuras de conexión a Internet y una falta de acceso a las nuevas tecnologías, en algunas familias y comunidades comparten teléfonos móviles entre varias personas, a lo que se suman las limitaciones en el acceso a la electricidad. Según observan en Radio Brahmaputra:

Los medios digitales dependen completamente de Internet (...) si vas a la zona rural entre las plantaciones de té, no encontrarás ninguna red de conexión allí. (...) Es 
muy difícil para ellos acceder a los principales medios de comunicación (...) también tienen un acceso limitado a Internet. (...) por lo que les es muy difícil saber lo que sucede en sus áreas (...) No tienen una plataforma de medios para saberlo." (...) Si hablamos de la comunidad marginada, principalmente están luchando por llevar adelante sus vidas, día a día, por sus recursos de subsistencia. (...) Lo principal es la participación, si no participan en este proceso, todos los días serán excluidos de este.

La participación de la audiencia es mayormente masculina en las radios comunitarias desde las que dicen apreciar que las mujeres oyentes de la radio no se atreven a hablar. Las dificultades percibidas por las entrevistadas hacen referencia a la participación activa de las mujeres en sus programas. En palabras de la participante de Gurgaon ki Awaaz:

... si un hogar tiene teléfono, la mujer escucha la radio (...) pero no llaman mucho porque sienten que los que llaman, muchos de ellos, son hombres y no se atreven a hablar. Pero cuando salimos y hacemos encuestas de campo y hablamos con los y las oyentes, las mujeres nos dicen que están al tanto de los programas de la radio.

Aunque aseguran que la participación ha ido en aumento en los últimos años a medida que ha ido creciendo el acceso de las mujeres a la telefonía móvil.

Ese cambio de los últimos años en el que de manera progresiva las mujeres han ido participando más en las emisoras, ha sido percibido igualmente en Radio Brahmaputra, donde explican que al principio las mujeres no participaban, no querían expresarse o responder a las preguntas de las reporteras. Pero han observado cómo de manera gradual han ido apropiándose de la emisora comunitaria y ahora no solo participan en entrevistas sino también como artistas en programas de radioteatro, acuden a la emisora en persona y participan en directo.

En referencia a las grabaciones en terreno, las entrevistadas aseguran que no es totalmente seguro para las mujeres por factores externos y que algunas mujeres del equipo han sufrido experiencias de discriminación, de relaciones de poder vividas durante su trabajo en la comunidad, así como un sentimiento de inseguridad por experiencias negativas durante la realización de este trabajo. La entrevistada de Radio Brahmaputra expresa: "A veces sienten que no es seguro cuando van a grabar en algunos lugares, es bastante inseguro para las mujeres".

Por otro lado, un factor que contribuye a generar un mayor interés por estos medios es el uso de los idiomas locales. Estas radios comunitarias son en algunos casos las únicas plataformas mediáticas que proporcionan un espacio en el que las comunidades pueden expresarse en sus propios idiomas, algunos son lenguas en peligro, como el bodo. Todo ello, además de contribuir a la identidad local y a la preservación de la diversidad lingüística local, también incide en la motivación e implicación de la comunidad en el proceso de la comunicación. Una reportera de Radio Bundelkhand explica que el factor que le animó a participar en la radio fue escuchar el idioma que hablaban en sus hogares, el bundeli, entonces acudió a la radio para expresar su interés en formar parte de la iniciativa.

Por último, la representación de las mujeres en las radios comunitarias analizadas está asegurada mediante una programación específica y enfocada en las mujeres. La producción de estos programas está en su mayoría financiada por agencias internacionales y departamentos gubernamentales formando parte de campañas de prevención y sensibilización. Entre las temáticas tratadas destacan las salud maternoinfantil, la nutrición y la salud sexual y reproductiva. Desde Gurgaon ki Awaaz, por ejemplo, aseguran que en sus programas intentan romper y abordar los roles de género.

En cuanto a los y las expertas colaboradoras de las radios, si bien en las emisoras no disponen de una agenda de contactos específica de mujeres expertas, en las tres radios aseguran contar con un número notable de mujeres expertas en su directorio, que han sido elegidas de acuerdo a las temáticas tratadas en las emisoras sin ser resultado de unas directrices formales. La participación de las mujeres expertas es calificada como relevante e indispensable para sus programas, dado que, según señalan, las expertas por el hecho de ser mujeres pueden tener una mayor empatía y ponerse en el lugar de las mujeres oyentes, comprender mejor las experiencias por las que pasan, incluidas situaciones de opresión, y responder de forma más adecuada a sus necesidades y expectativas, tal y como afirma la participante de Gurgaon ki Awaaz: "Elegimos mujeres expertas porque, al ser ellas mismas mujeres, pueden comprender mejor las experiencias por las que pasan las oyentes".

\section{Conclusiones}

Las dificultades evidenciadas en este contexto para lograr mayores derechos de comunicación para las mujeres, así como las transformaciones de esta realidad manifiestas, muestran la necesidad de incluir aprendizajes desde las experiencias particulares de las mujeres participantes de las radios comunitarias en la investigación en Comunicación Participativa.

Entre los principales aportes de la investigación, los datos empíricos del trabajo de campo ofrecen información del contexto y de las dinámicas de poder desde las experiencia particulares de las mujeres participantes. De este modo, hemos podido conocer las vivencias de las comunicadoras de las radios comunitarias estudiadas a través de sus relatos y testimonios. 
A partir de los datos etnográficos recopilados podemos dar continuidad a la investigación con la búsqueda de conexiones con otros contextos y oportunidades de réplica de buenas prácticas y análisis comparativos que continúen indagando en la identificación de estrategias que contribuyan a la realización del derecho a la comunicación de las poblaciones subalternas y a una mayor participación y peso de las mujeres como comunicadoras.

Como conclusión, los resultados obtenidos confirman que desde las experiencias de las radios comunitarias es posible encontrar respuestas a las problemáticas del silenciamiento, e identificar posibles vías para romper las dinámicas estructurales de la exclusión.

Las experiencias mediáticas documentadas están involucrando en este proceso a poblaciones excluidas y silenciadas por las lógicas de poder. Son, por tanto, respuestas originales enfocadas en la transformación de las dinámicas estructurales de exclusión y silenciamiento. Igualmente, estos casos de estudio evidencian la necesidad de los grupos subalternos de la realización de su derecho a la comunicación.

Frente a las problemáticas mencionadas de la exclusión mediática, la brecha digital y el silenciamiento de las voces subalternas, las experiencias de las participantes destacan la contribución de los medios comunitarios a la transformación de estas realidades y muestran posibles caminos hacia la democratización del acceso a los recursos, incluidos el conocimiento y la información.

En definitiva, desde su crítica inicial a las problemáticas de exclusión existentes y mediante su capacidad de acción, las mujeres comunicadoras y las radios comunitarias tratan de construir, con su trabajo, espacios en los que las poblaciones subalternas puedan pasar de ser silenciadas a expresarse libremente y en los que poner en práctica su derecho a la comunicación, trazando un camino desde la exclusión social a la expresión popular.

\section{Notas al final}

Este trabajo presenta algunos de los resultados de la tesis doctoral de la misma autora (2019). Las ideas principales del análisis fueron presentadas como comunicación en el XII Congreso Internacional de Ciberperiodismo (Bilbao, 9 y 10 de noviembre de 2020).

\section{Referencias}

AMARC WIN. (2008). Gender Policy for Community Radios. Quebec. http://www.isiswomen.org/downloads/GenderPolicy/GP4CR_ English.pdf

AMARC WIN Asia-Pacific. (2013). Women in Community Radio in Asia Pacific. A comparative report of the AMARC-WIN AP survey in 2013 and
2006.

Chatterji, S. (2010). Information as Empowerment, en Sharma, K. (Ed.), Missing half the story. Journalism as if Gender Matters (pp. 272-257). Zubaan.

Drèze, J., y Sen, A. (2013). Democracia, desigualdad y razonamiento público, en Una gloria incierta: India y sus contradicciones (pp. 271-304). Taurus.

Dutta, M. J. (2012). Voices of Resistance: Communication and Social Change. Purdue University Press.

Freire, P. (1970). Pedagogía del oprimido. Tierra Nueva.

IAMAI, y NIELSEN. (2019). Digital in India. Round 2 report. IAMAI Nielsen.

Jeffrey, R. (2001). [NOT] being there: Dalits and India's newspapers. South Asia: Journal of South Asian Studies, 24(2), 225-238. https:// doi.org/10.1080/00856400108723459

Kaplún, M. (2002). Una pedagogía de la comunicación (el comunicador popular). Caminos.

Kishor, S., y Gupta, K. (2009). Gender Equality and Women's Empowerment in India.2005-06 National Family Health Survey (NFHS-3). Mumbai.

Krohling Peruzzo, C. M. (2016). La comunicación en los movimientos sociales y el derecho a la comunicación: señales de un derecho de ciudadanía de quinta generación. Commons. Revista de Comunicación Y Ciudadanía Digital, 5(2), 8-35. Recuperado de https://revistas.uca. es/index.php/cayp/article/view/3234

MacBride, S. (1980). Many voices, one world. UNESCO.

Malik, K. K. (2012). Community Radio: eliminating voice poverty. recperado. The Hoot.org. de http://www.thehoot.org/story_popup/ community-radio-eliminating- voice-poverty-5739

Malik, K. K. (2015). Our media, our principles. Journalism Studies, 1-14. https://doi.org/10.1080/1461670X.2015.1054195

Malik, K. K., y Bandelli, D. (2012). Community Radio and Gender Towards an Inclusive Public Sphere. India Media Symposium: Public Spheres, the Media y Social Change in India. Queensland.

Mander, H. (2015). Looking Away: Inequality, Prejudice and Indifference in New India. Speaking Tiger.

Mohanty, R. (2010). Contesting development, reinventing democracy: grassroots social movements in India, en Thompson, L., y Tapscott, C. (Eds.), Citizenship and social movements: perspectives from the global South (pp. 239-259). Zed Books.

Moitra, A., y Kumar, A. (2016). Hill Women's Voices and Community Communicationabout Climate Change: The Case of Henvalvani Community Radio in India, en Servaes, J., y Oyedemi,T. (Eds.). The Praxis of Social Inequality in Media (pp. 137-160). London: Lexington Books.

Naqvi, F. (2007). Waves in the hinterland: the journey of a newspaper. NZubaan, Nirantar.

One World Foundation India. (2016). Community Radio in India. Towards Diversity y Sustainability. Community Radio Compendium -2016. New Delhi.

Pande, R. (2012). Gender Gaps and Information and Communication Technology. A case study from India, en Pande, R., y van der Weide, T. P. (Eds.). Globalization, Technology Diffusion and Gender Disparity. Social Impacts of ICTs (pp. 277-291). IGI Global. https://doi. 
org/10.4018/978-1-4666-0020-1.ch022

Pavarala, V., y Malik, K. K. (2007). Other Voices: The Struggle for Community Radio in India. Sage.

Rodriguez, C. (2001). Fissures in the Mediascape. An International Study of Citizens' Media. Hampton Press.

Roy, A. (2016). Determining destinies. Building transparency and accountability through citizen involvement, en Mudgal, V. (Ed.), Claiming India from below: Activism and democratic transformation (pp. 261-291). Routledge.

Skuse, A., Fildes, J., Tacchi, J., Martin, K., y Baulch, E. (2007). Poverty and digital inclusion: Preliminary findings of Finding a Voice Project. UNESCO

Spivak, G. C. (1998). ¿Puede hablar el sujeto subalterno? Orbis Tertius III(6), 175-235

Tacchi, J. (2009). Finding a voice : digital storytelling as participatory development inSoutheast Asia, en Hartley, J., y McWilliam, K. (Eds.). Story circle : digitalstorytelling around the world. Wiley-Blackwell.

Tacchi, J., Foth, M., y Hearn, G. (2009). Action research practices and media for development. International Journal of Education and Development Using ICT, 5(2), 32-48. https://www.learntechlib. org/p/42319

Thomas, P. N. (2011). Negotiating Communication Rights: Case Studies from India. Sage Publicactions. https://doi. org/10.4135/9781446270325

Thomas, P. N. (2014). Public hearings and public spheres in India: the case of the right to information (RTI) movement. Media International Australia. Public Spheres and the Media in India, (152), 133-142.
Goldsmith, B., y Beresford, M. (26 de junio de 2018). The world's most dangerous countries for women 2018. Thomson Reuters Foundation. https://www.reuters.com/article/women-dangerous-poll-idINKBN1JM076

Yalala, N. (2015). The Role of Community Radio in Empowering Women in India. Journal of Mass Communication y Journalism, 5(2), 1-4. https://doi.org/10.4172/2165-7912.1000245

CV

Amaia Landaburu. Es doctora con mención internacional y cum laude por la Universidad de Málaga en el programa de doctorado en Educación y Comunicación Social. Es licenciada en Ciencias de la Comunicación por la Universitat Autònoma de Barcelona y Máster en investigación en Comunicación Periodística por la Universidad de Málaga. Fue beneficiaria de una ayuda predoctoral de movilidad internacional otorgada por la Universidad de Málaga para la realización de tesis con mención internacional que le permitió realizar una estancia doctoral de cuatro meses en el Centro MICA (EA4426) de la Universidad Bordeaux-Montaigne, Francia. Previamente, realizó otra estancia de investigación de doce meses en el Centro CCMG de la Universidad Jamia Millia Islamia en Nueva Delhi, India. También cuenta con publicaciones en revistas científicas de revisión por pares y ha participado en congresos internacionales. Actualmente es investigadora contratada en la Universidad del País Vasco/Euskal Herriko Unibertsitatea.

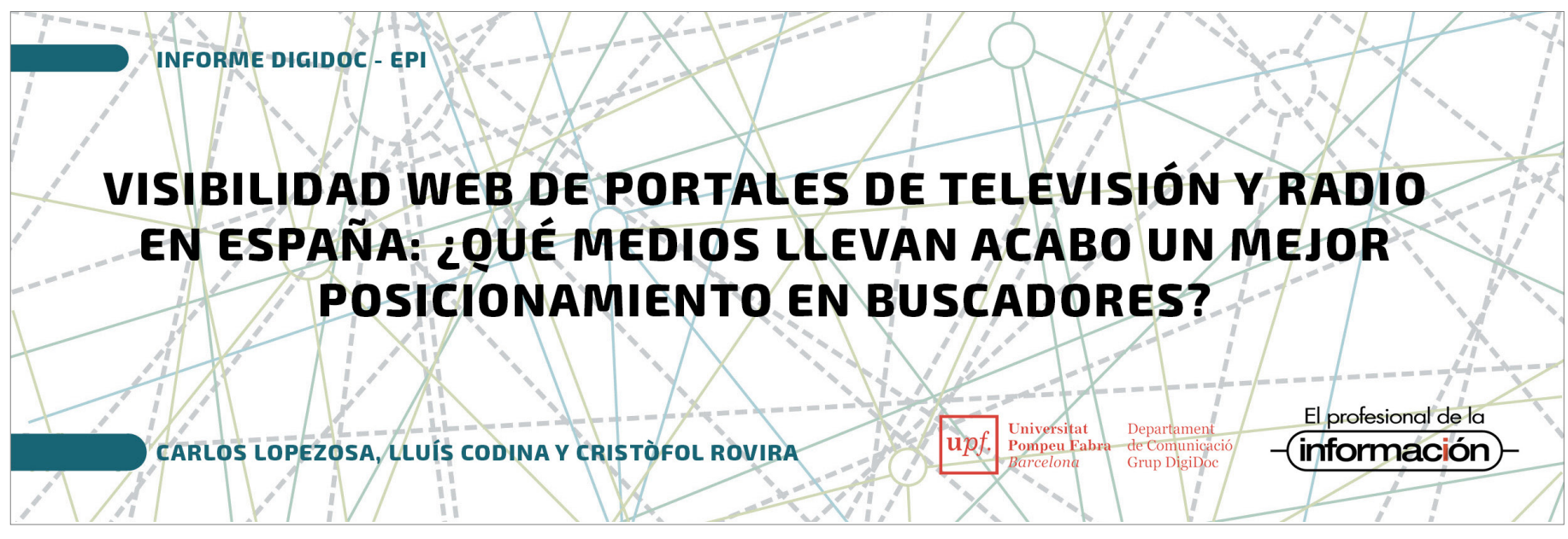

\section{Observatorio de Cibermedios}

\section{https://observatoriocibermedios.upf.edu/}
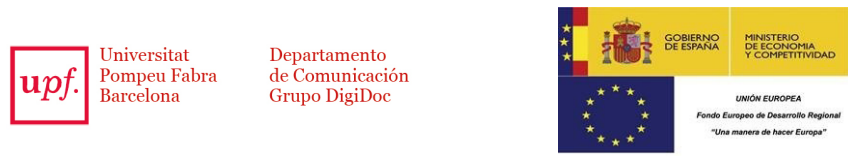

El Observatorio de Cibermedios es una producción del Grupo de Investigación en Documentación Digital y Comunicación Interactiva (DigiDoc) del Departamento de Comunicación de la Universitat Pompeu Fabra.

El Observatorio de Cibermedios (OCM) forma parte del proyecto del Plan Nacional "Narración interactiva y visibilidad digital en el documental interactivo y el periodismo estructurado". RTI2018-095714-B-C21 (MINECO/FEDER), Ministerio de Ciencia, Innovación y Universidades (España). 